\title{
Link Available Bandwidth Monitoring for QoS Routing with AODV in Ad Hoc Networks
}

\author{
Stephane Lohier ${ }^{1}$, Yacine Ghamri Doudane ${ }^{2}$, and Guy Pujolle ${ }^{1}$ \\ ${ }^{1}$ LIP6 - University of Paris VI, 8, rue du Capitaine Scott, 75015 Paris - France \\ ${ }^{2}$ IIE-CNAM, 18, allée Jean Rostand, 91025 Evry Cedex - France \\ stephane.lohier@lip6.fr, ghamri@iie.cnam.fr, \\ guy.pujolle@lip6.fr
}

\begin{abstract}
Due to bandwidth constraint and dynamic topology of mobile ad hoc networks, supporting Quality of Service is a challenging task. In this paper we present a solution for QoS routing based on an extension of the AODV reactive routing protocol that deals with bandwidth monitoring. The solution uses an IEEE 802.11 MAC layer as the underlying technology and the QoS routing decision is based on simple but accurate measurements, at the MAC layer, of the available bandwidth on each link of the route. In addition, to allow a QoS loss recovery, a notification mechanism is used to inform the source about bandwidth degradation on a link. This reactive solution using standard protocols is adapted to small and dynamic ad hoc networks. A complete simulation set shows that, with the proposed QoS routing protocol, bandwidth on a route is significantly improved without overhead.
\end{abstract}

Keywords: QoS routing, ad hoc, AODV, IEEE 802.11.

\section{Introduction}

Throughputs reached today by mobile ad hoc networks based on the IEEE $802.11 \mathrm{~b}$ and 802.11g standards [1-2] enable the execution of complex applications (video conference, distribution of multimedia flows...). However, these applications consume significant amounts of resources and can suffer from an inefficient and unfair use of the wireless channel. Therefore, new specific QoS solutions need to be developed taking into account the dynamic nature of ad hoc networks. Since these networks should deal with the limited radio range and mobility of their nodes, we believe that the best way to offer $\mathrm{QoS}$ is to integrate it in routing protocols. These protocols will have to take into consideration the QoS required by the applications, such as bandwidth constraints, in order to select the adequate routes.

In our current work, a new available bandwidth monitoring technique is proposed. This monitoring is integrated to the routing process. It acts on each link allowing determining if a particular bandwidth demand can be granted or not. In addition, bandwidth monitoring allows detecting, after the QoS route is traced, if the available bandwidth is degraded on a particular link and thus leading to QoS loss on the route using the concerned link. In that case, a QoS loss recovery mechanism is triggered to inform the source which will be able to start a new QoS route search. 
The QoS routing process is based on the AODV (Ad hoc On-demand Distance Vector) routing protocol [3] and uses QoS extensions proposed by the originators of AODV [4]. To that first proposal, we add the bandwidth measurement and the QoS loss recovery mechanism. The choice of a standard and reactive routing protocol such as AODV is justified in small, highly dynamic and mobile ad hoc networks. Indeed, it is proved that reactive protocols are well suited in this kind of networks [5-6] which respond to the most common use cases, such as meetings or emergencies, where the number of nodes is limited and variable in a reduced space.

Furthermore, the bandwidth measurements are realized according to 802.11 operations without influencing them. Theses measures are thus passive and compatible with the reactive routing process. Thus, our solution uses recognized and standard protocols without modify them: no modification of the 802.11 standard for the measurements and QoS routing extensions for AODV without additional signalling.

The rest of the paper is organized as follows. In section 2, we introduce the different solutions for bandwidth monitoring in ad hoc networks and the selected one. Section 3 describes the QoS routing process. Section 4 concerns the QoS loss recovery mechanism. Section 5 presents the simulation results. Finally, section 6 concludes the paper and presents some perspectives.

\section{Bandwidth Measurement}

Before describing the different solutions for the bandwidth measurement, let us clarify some important preliminary points in an ad hoc context and with a reactive routing protocol:

- As the objective is to establish a QoS route between the nodes, the available bandwidth $(a b w)$ must be monitored for each link of the route rather than in the neighbourhood of each node.

- For a given source node A, the estimate on the link can be different according to the receiving node $\left(a b w_{A B} \neq a b w_{A C}\right)$. Indeed, the receivers do not have the same neighborhood. Moreover the estimate is not symmetrical because the transmitter and receiver nodes do not have the same neighborhood $\left(a b w_{A B} \neq a b w_{B A}\right)$.

- To estimate $a b w_{A B}$ on the link between A and B, it is necessary to take into account the bandwidth consumed by A and B neighbors.

- It is also necessary to take into account the disturbances caused by the nodes in the interference zone of the receiver B (it is generally considered that the interference zone has a ray double of that of the neighbourhood zone). Indeed, the transmissions of a node in the interference zone of B may cause an important decrease in the Signal to Noise Ratio (SNR), leading to frame losses in the vicinity of B.

Then, the different studies present in the literature can be classified according to the layer where they act: network or link.

At the network layer, the study [7] presents an estimation method, named hello method, which is very close to that proposed in the BRuIT protocol [8], but with its integration into the routing process. The principle is that a node can estimate its own available bandwidth when receiving "hello" probes containing information on the 
bandwidth consumed by its one hop neighbours (two hops for BRuIT protocol). The principal disadvantage of these type of solutions is that they require an important control traffic, particularly for the messages exchanges intended to maintain important and static routing tables, which gives a proactive character on the QoS routing process, more adapted to dense and not very mobile ad hoc networks. In addition, in [7], the "QoS-aware" approach is slightly different from our insofar as the aim is to preserve the end to end average throughput, compared to the solution without QoS, and not to guarantee the throughput required by a source.

At the link layer, most of proposed solutions [9-11] are based on the calculation of the medium availability ratio for each node by measuring the idle and/or the activity periods at the MAC layer. The main disadvantage of these methods is the need for emitting systematically a consequent number of measurement packets for each estimate, which is not appropriate if we want to keep the reactive nature of the routing strategy. In addition, the method is intrusive as it disturbs the neighbouring flows and thus has an influence on the value which it is supposed to measure.

Finally, the constraints related to the selected context (reactive routing, bandwidth on a link, neighborhood zone and interference zone) show us that the estimates are more relevant at the MAC layer, even if the measure is not passive. Indeed, the consideration of neighboring or interfering flows is intrinsically related to measurement and does not require implementing complex signaling overhead at the network layer, which would be in opposition to the reactive character of the routing.

The various solutions at the MAC layer are all based on an initial measurement of the activity or idle periods of the radio channel. These durations are not directly available on an 802.11 interface but can be measured simply by introducing measurement packets provided with timestamps. It is the method employed in [10] and [11]. We include in our proposal this method which takes directly into account the effects of neighbouring and interfering flows. To this method, we add a correction on the packet size, a mechanism allowing to alert the source of an increase in the bandwidth on the link and we avoid the systematic use of a measurement window, conflicting to the reactive character.

Let us detail the measurement principle at the MAC layer on a link between two stations A and B. The various stages of transmission on the 802.11 channel are summarized on Fig. 1. We are interested here in the duration of the channel occupancy and in the duration $d_{A B}$ between the moment when the packet is ready to be emitted by $\mathrm{A}$ at the MAC layer and the moment when the packet is received by B (i.e. the moment when the MAC acknowledgement is received by $\mathrm{A}$ ).

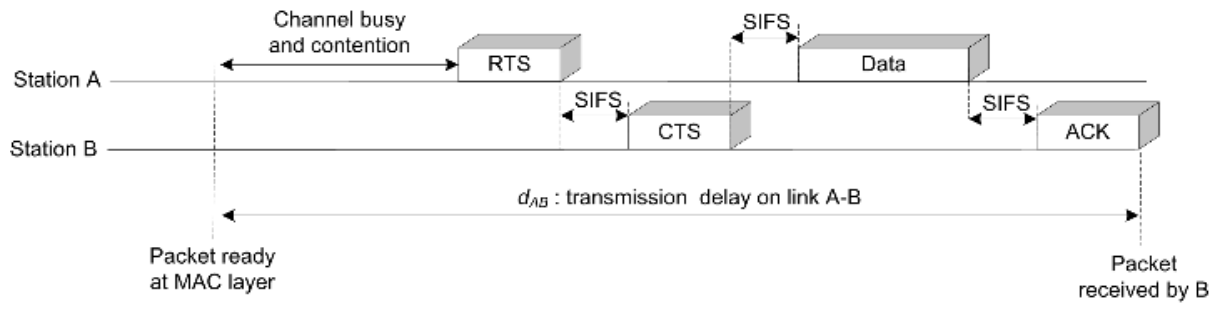

Fig. 1. Transmission stages on the 802.11 channel 
A first estimate of $a b w$ is carried out at the sender side by calculating the relationship between the size of the measurement packet and the $d_{A B}$ duration necessary to its transmission on the channel:

$$
a b w=\frac{\text { Data_length }}{d_{A B}}
$$

We can split up $d_{A B}$ into a variable part and a constant part. The variable part depends on the channel occupancy and on the duration of the contention window. The constant part corresponds to the transmissions of the control and data frames when station $\mathrm{A}$ is in emission phase:

$$
a b w=\frac{\text { Data } a_{-} \text {length }}{T_{B U S Y}+T_{C W}+T_{C S T}}
$$

The constant term $T_{C S T}$ shows dependence with the data packets's size $\left(t_{M P D U}\right)$ :

$$
T_{C S T}=t_{R T S}+t_{C T S}+t_{M P D U}+3 t_{S I F S}+t_{A C K}+4 t_{P H Y}
$$

The duration $T_{B U S Y}$ which corresponds to the sequence of the various NAV (Network Allocation Vector) timers imposed by the stations in emission, until the station has the right to emit, is directly a function of the traffic in the neighbourhood and interference zones. The duration of the contention window $T_{C W}$ is related to the backoff algorithm of 802.11 standard, it is thus a partly random duration but also related on the contentions and the retransmissions and consequently also dependent on the number of neighbouring and interfering flows.

We thus verify that in theory, this first simple estimate of the available bandwidth on the link is related to the sender's and receiver's neighbouring flows (if the contentions increase, $T_{B U S Y}$ and $T_{C W}$ increases and $a b w$ decreases) and also to the flows in the interference zone of the receiver (if the number of retransmissions caused by losses or errors increases, the MAC acknowledgements will be delivered less quickly and $T_{C W}$ will increase).

The QoS decision must be made, for each link, on the receiving node (see next section). A timestamp can thus be transmitted by the transmitting node in a request or data packet's extension to indicate to the receiver the moment when the packet is ready at the MAC layer.

Furthermore, this first approximation depends on the packet's size and it is necessary to carry out a correction to take into account the real data packet's size and not the RREQ packet's length, when those are used for measurement. We thus propose a correction on the $d_{A B}$ duration:

$$
d_{A B}^{\prime}=d_{A B}+\frac{\left(\text { Data_length }-R R E Q \_ \text {length }\right)}{\text { Throughput }}
$$

We can then express the corrected bandwidth:

$$
a b w^{\prime}=\frac{\text { Data_length }}{d_{A B}+\frac{\text { Data_length }- \text { RREQ_length }}{\text { Throughput }}}
$$


To preserve the reactive feature of our QoS routing and to avoid intrusive measurements, we choose to not use beforehand, as in the studies [10-11], a temporal window to calculate an average on successive measures with, for example, "hello" packets. In our proposal, the estimate of the available bandwidth is initiated by the first request packets charged to trace the QoS route but uses indifferently all the control or data packets having already crossed the concerned link before the request (passive measurement). A first order smoothing taking into account the previous measurements realized with the request or data packets is then realised. We can thus express the available bandwidth:

$$
\widehat{a b w}(t)=\alpha \cdot a b w(t)+(1-\alpha) \cdot a b w(t-1)
$$

Moreover, to guarantee the validity in time of the measure and to take into account the arrival or the extinction of neighbouring or interfering flows, we propose a mechanism integrated into the routing protocol which informs the source of a reduction of $a b w$ beyond a preset threshold (see section 4).

Another limitation of our estimate is not specific to the chosen method but more generally related to admission control on a radio medium. Indeed, it is impossible to estimate beforehand the effect, due to the contentions on the medium that a new data flow will have on the available bandwidth measured according to the flow already present in the neighbourhood or interference zones. Thus the admission of a new flow based on the preliminary estimate, the least intrusive possible, will cause more contentions and an inevitable fall of the available bandwidth for all flows. To overcome this issue, one of the usual solutions is to introduce a margin into the admission control to compensate the global reduction in bandwidth (see next section). This solution can be improved with a dynamic re-negotiation when the load in the neighbourhood is modified.

\section{QoS Routing Process}

The QoS routing objective is to find a route with enough available resources to satisfy a QoS request. Thus, a source having a bandwidth constraint transmits a RREQ packet with QoS bandwidth extension (an optional extension is foreseen by AODV for its main packets RREQ and RREP). This extension indicates the minimum bandwidth that is needed on the whole path between the source and the destination. Before forwarding the RREQ packet, an intermediate node compares its available bandwidth $a b w$ (note that $a b w$ is estimated for the concerned link and not for the node receiving the RREQ) to the bandwidth field indicated in the QoS extension (see Fig. 2). If the bandwidth required is not available (i.e. if according to the estimate it does not remain sufficient bandwidth on the concerned link), the packet is discarded and the process stops.

As specified previously, even if the traffic used for measurement does not affect significantly the entire traffic load during the route search, it is necessary to introduce a margin on the comparison to compensate the effect of the new flow on other flows when the route will be established (new contentions will reduce the estimate of the inactivity periods of the channel). For example, for a QoS request of 500kbps, a value of 540kbps for $a b w$ on one of the links will be insufficient if the selected margin is 
$10 \%$ of the request. This admission control margin has to be tuned according to the traffic characteristics and the density of the network.

In response to a QoS request, the destination sends a RREP packet with the available bandwidth measured on the link which precedes it. Each intermediate node receiving the RREP compares the bandwidth field of the extension with the available bandwidth on the link which precedes it and keeps the minimum between these two values to propagate the RREP on the selected route (Fig. 2). The received value (bandwidth field of RREP) is also recorded in the temporary routing table for the concerned destination. It indicates the minimum available bandwidth for the destination. This entry update allows an intermediate node to answer the next RREQ simply by comparing the minimum bandwidth fields of the table with the value of the transmitted extension (the request does not need to be forwarded toward the destination). This information remains valid as long as the route is valid (in AODV, the lifetime is based on sequence number transmitted in control packets and on the "ACTIVE_ROUTE_TIMEOUT" parameter).

Finally, one should note that the data packets are also used to estimate $a b w$ and to calculate an average value. This is realized in order to assess the validity in time of link bandwidth measurement. Also, in the case where a particular link is already used by a route, this continuous estimate will serve during new QoS route search involving the concerned link.

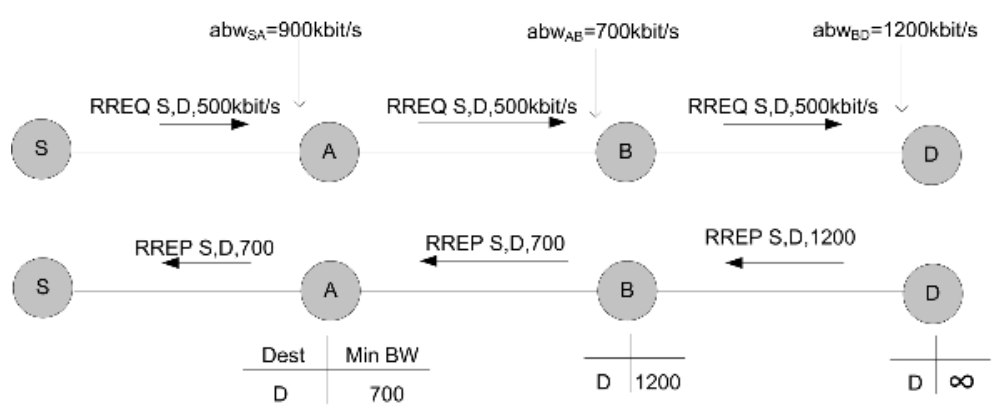

Fig. 2. Example of QoS bandwidth request and response

\section{QoS Loss Recovery Mechanism}

When because of node movement or new traffic arrival, the available bandwidth decreases on a link of the route, it is possible that the QoS constraint required by the source can not be respected any more. In order to overcome that, a QoS loss recovery mechanism is implemented. The latter uses a predefined QoS Bandwidth Margin (QBM). A route error packet (RERR) is generated when an intermediate node detects a decrease in $a b w$ that is greater than QBM. As for standard AODV route error mechanism, the RERR packets are sent to all the precursors stored for all the concerned routes. These routes are then erased from routing tables. When the RERR packet reaches the QoS source, it initiates a new route discovery with RREQ packet if the QoS route is still needed. 
In the example of figure 3 , the link between node $\mathrm{A}$ and node $\mathrm{B}$ is used for two QoS routes: S1-C-A-B-D1 and S3-A-B-F-D3. When B receives a RREQ packet from A for the QoS route from S1 to D1 (or for any other QoS route discovery involving the link of A towards B), it compares its previous value of $a b w$ with the new measured one. Then, if it detects a decrease in $a b w$ that is greater than QBM, the node $\mathrm{B}$ send a RERR packet to A which specifies the list of the unreachable destinations (D1 and D3). After reception, node A forwards the RERR packet to all the precursors (C and S3) stored in its routing table for the routes to destinations D1 and D3 and then erases these routes.

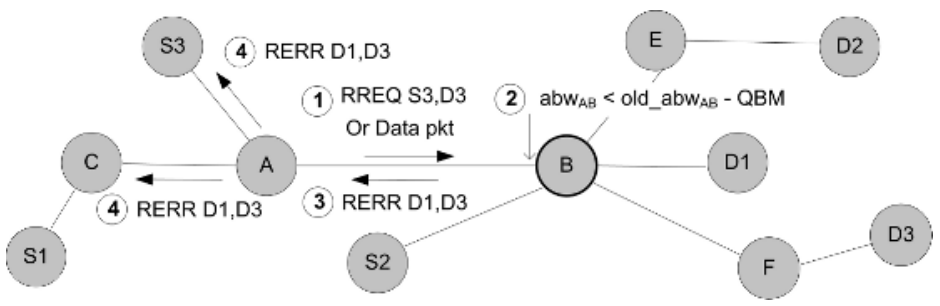

Fig. 3. Example of QoS Bandwidth lost

Note that the $a b w$ is measured each time a RREQ or a data packet is received by a node, which generally corresponds to a change of the traffic load (new source) or of the network topology (node movement and route failure) producing a possible loss of QoS. Furthermore, if the QBM margin is selected too large, the source node will not be informed of an eventual QoS loss. Conversely, if the margin is too small, useless RERR packets can be generated, causing new RREQ broadcasts. This undesirable control packet transmission induces an undesired overhead, slowing down data packet exchanges, even if the QoS constraint is initially respected. So, an adequate choice of this margin, according to the characteristics of the network, is necessary.

\section{Simulation Results}

In order to evaluate the performance of our QoS routing protocol, we simulate the proposed mechanisms using NS-2 [16] and its 802.11b extensions.

The radio model allows a signal rate of $11 \mathrm{Mbps}$, a transmission range of $100 \mathrm{~m}$ and a detection range of $200 \mathrm{~m}$. These ranges correspond to the neighbourhood and interference zones previously described and are close to the typical values obtained for an indoor $802.11 \mathrm{~b}$ deployment. The number of mobile nodes is set to 10,20 or 50 nodes giving three simulation sets. These nodes are spread randomly in a $400 \times 400 \mathrm{~m}$ area network and they move to a random destination every 30 s with a speed randomly chosen between 0 and $2 \mathrm{~m} / \mathrm{s}$ (maximal speed for a pedestrian user). Simulations run for 300 s.

Traffic sources are CBR (Constant Bit Rate). Their rates correspond to the QoS constraint (i.e. the bandwidth required by the source for its QoS application) and are fixed to $500 \mathrm{kbps}$ (122 packets of 512 bytes per second). This value is selected to reach the saturation limits (more than $50 \%$ of the delivered packets are not received) 
when the number of sources is maximum. Several simulations are realized by varying the number of CBR sources from $10 \%$ to $100 \%$ of the total number of nodes (a node can integrate several CBR sources). Preliminary measures not represented here made it possible to fix optimal values for the different parameters: 0.2 for the smoothed factor $\alpha$ used for $a b w$ estimation; 150kbps (30\% of the request) for the admission control margin on the bandwidth requests and 50kbit/s (10\% of the request) for QBM. For the various network densities, this value of QBM margin corresponds to the best compromise between the reactivity and the overhead and thus gives the best results in terms of global average throughput and number of transmitted packets.

Figure 4 and 5 present the average throughput on all routes when data packets are sent from a source to a destination. The throughput is evaluated at the destination by measuring the rate of the received packets during the connection periods (when the CBR source is active and the route between the source and the destination is up).

For low density networks (Fig. 4), the bandwidth constraint is almost respected with a fall of $1 \%$ on a 20 -node network (minimum value of $495 \mathrm{kbps}$ for $100 \%$ of sources) and of $3 \%$ on a 10 -node network (minimum value of $484 \mathrm{kbps}$ for $60 \%$ of sources). The results are slightly better with 20 nodes because the compromise between the density of the network and the traffic load is much favourable to search and maintain QoS routes in this case. Without QoS routes, the available bandwidth on the links is not considered and the delay between two packet's receptions on the selected route can increase significantly, thus reducing the average global throughput (from 500kbps to $420 \mathrm{kbps}$ for 20 nodes and $100 \%$ of sources).

For a high density network of 50 nodes, the improvement is still better (Fig. 5). With QoS, the throughput is always higher than 470kbps $(6 \%$ less compared to the constraint). When the number of QoS sources reaches $100 \%$, the average global throughput slightly decrease (440kbps) because of the transitional periods corresponding to the route reorganizations are more frequent. Without QoS, the throughput is not controlled at all and it decreases very quickly: less than $250 \mathrm{kbps}$ (50\% of the constraint) as soon as the number of sources becomes higher than $20 \%$.

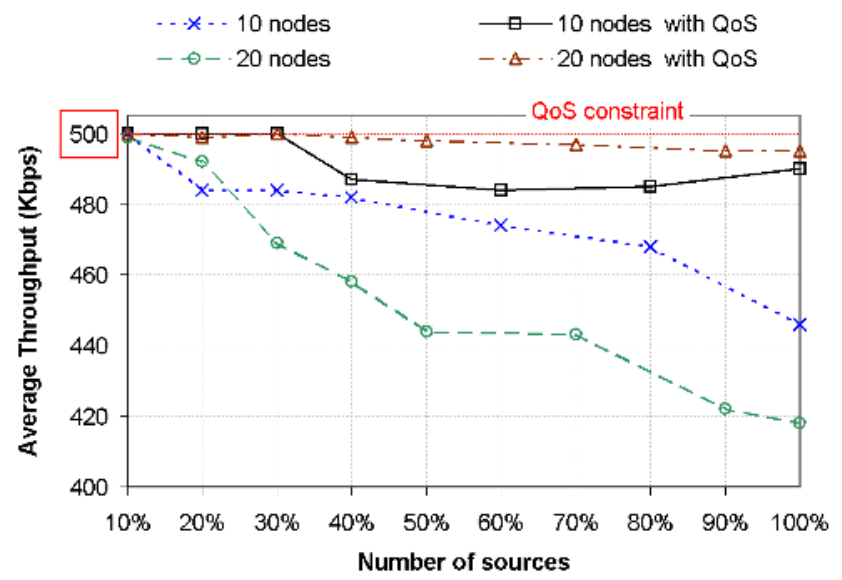

Fig. 4. Average Throughput / Number of sources (10 and 20 nodes) 


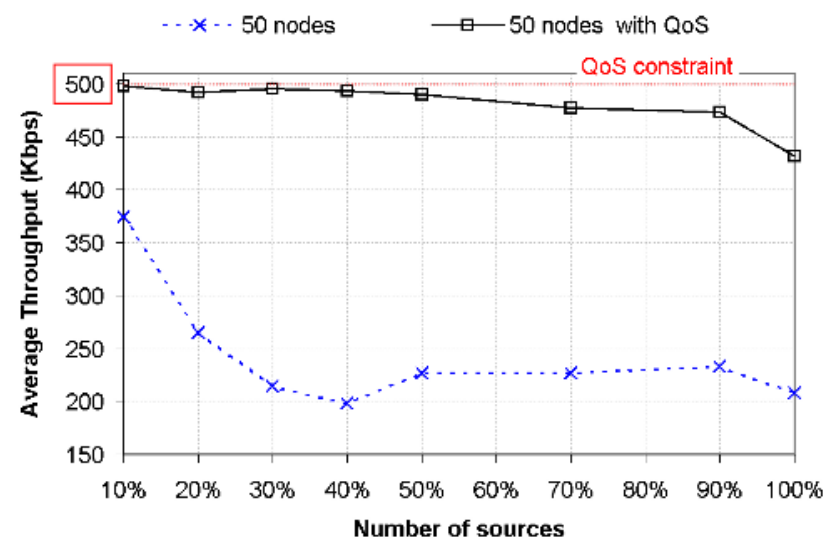

Fig. 5. Average Throughput / Number of sources (50 nodes)

Figure 6 shows the evolution of the number of packets which are dropped along the way when the number of sources increases. The y-axis thus represents the ratio between the number of packet dropped by an intermediate node (whatever the reason: mobility or contention) and the number of data packet sent by the source when a route is supposed to exist. In all the cases, the results are clearly better with QoS: less than $12 \%$ of dropped packets whatever the density of the network. These important improvements are due principally to two factors: the rejection of the unreliable routes during the QoS route setup procedure and the QoS loss notifications mechanism which avoid sending packet when a high contention risk is foreseen. Note that without QoS, the number of dropped packets can be very important (up to $86 \%$ with 50 nodes), confirming that the accepted routes are not reliable.

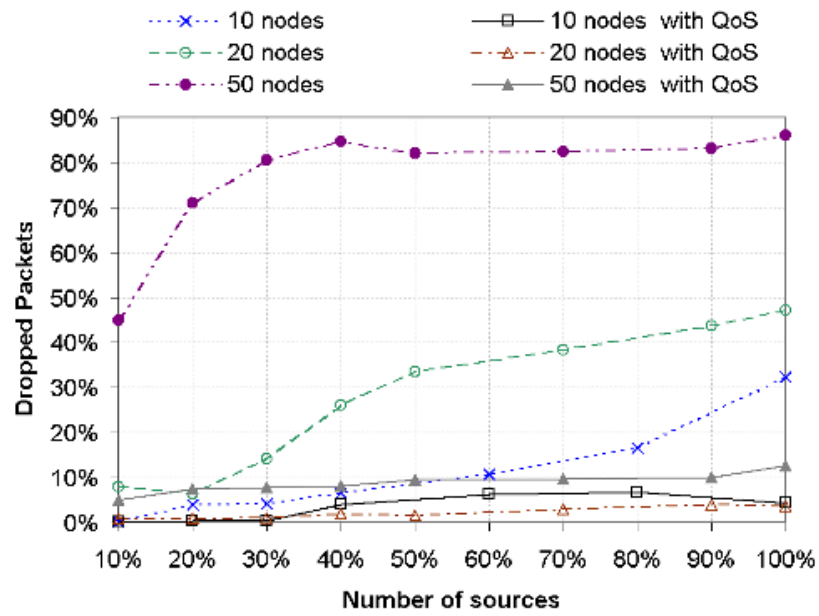

Fig. 6. Packets Dropped on the way / Number of sources 
Figure 7 presents the evolution of the total number of undelivered packets according to the number of sources. The y-axis thus represents the ratio between the number of packets generated by the CBR sources which never reach their destinations (whatever the reason: no route at the source, mobility or contention) and the total number of packets generated by the CBR sources. Let us note that this latter is about identical with or without QoS, which thus makes it possible to compare, with theses curves, the absolute number of undelivered packets or, by difference, the absolute number of received packets.

We notice that the number of undelivered packets is not very different with or without QoS routing (it is even better with QoS in a 50-node network). This result shows that most of the undelivered packets are rejected at the source because no route is possible or even are dropped along the way because of mobility rather than because the available bandwidth is insufficient on the crossed links. Furthermore, we can notice that the number of received packets, obtained by subtraction, is also quite close with QoS but for all these received packets, the QoS condition is met (remember that without QoS the average throughput can be less than 250kbps). This show that the QoS routes are not obtained to the detriment of the number of delivered packets.

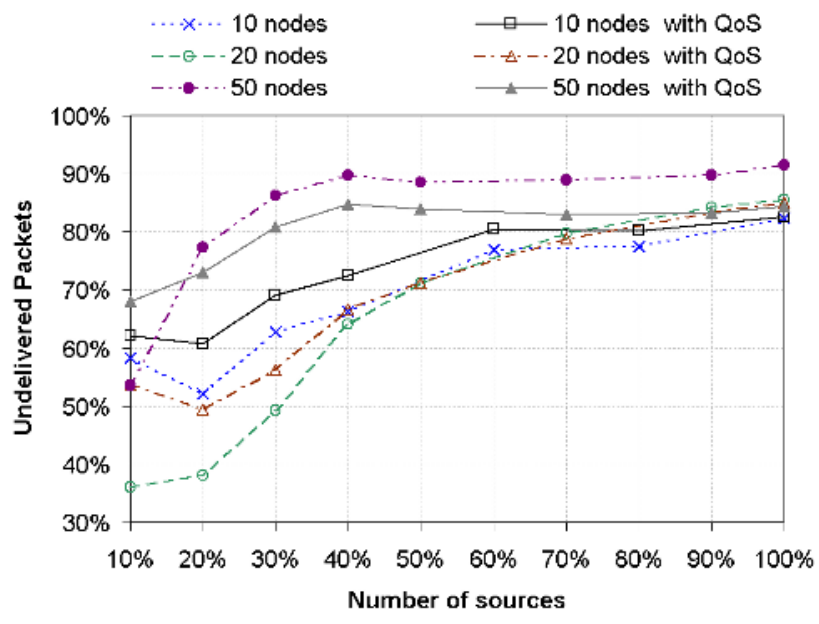

Fig. 7. Undelivered CBR Packets / Number of sources

Figure 8 shows the effective packet overhead due to the AODV control messages. The y-axis thus represents the ratio between the number of AODV packets and the number of AODV packets plus the number of received data packets. We can observe that for 50-node network, even if there are more AODV packets which circulate with the QoS solution, these packets are much more useful to find a reliable route and to avoid the losses. For 10-node and 20-node networks the difference is very weak showing here also, if we take into account the bandwidth improvements (Fig. 4), that the QoS overhead is effective. 


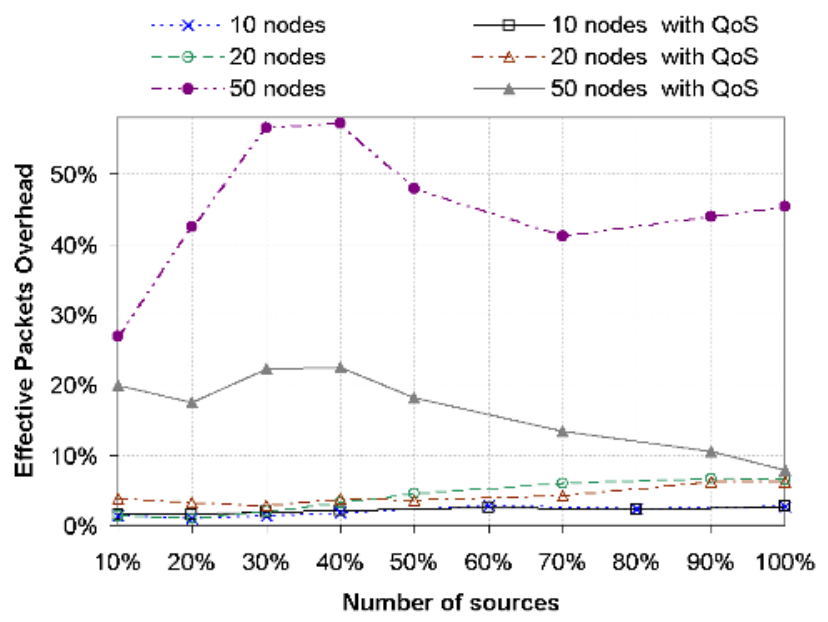

Fig. 8. Effective Overhead / Number of sources

\section{Conclusion}

In this paper, we proposed and evaluated a QoS routing solution for ad hoc networks based on link available bandwidth monitoring. This solution uses standard protocols (AODV for the routing process and 802.11 at the link layer) and is intended for small and dynamic ad hoc networks in which an important reactivity is necessary. The QoS routes are traced node by node and the proposed routing algorithm uses only extensions of the AODV request (RREQ) and reply (RREP) packets. The bandwidth measurement is initiated on RREQ arrivals in a node (these times correspond to a network state change: arrival of a new flow or route recovery following a node movement) to avoid increasing the overhead unnecessarily. Moreover, a QoS loss recovery mechanism, also based on the existing extensions, is used to take into account the variations of the available bandwidth due to the dynamic nature of the ad hoc network and its traffic.

The proposed QoS routing with QoS loss recovery gives very satisfying results. The required bandwidth on the QoS routes is obtained with less than 6\% of fall in almost all the cases. Comparatively, this bandwidth that the source nodes seek to maintain thanks to the QoS routing can be divided by two in the absence of this one. In addition, these improvements are not obtained to the detriment of the number of routed packets since in all the cases, the relative rate of dropped packets is lower with the QoS solution. Lastly, the effective overhead is less important with the QoS constraint whatever the density of the network.

As for future works, we are targeting the issue of the dynamic optimization of QDM and the admission control margin. A first idea will be to analytically derive a relationship between these margins and both the number of flows crossing each node and/or their bandwidth/delay requests. 


\section{References}

1. IEEE 802.11 WG, Part 11: Wireless LAN Medium Access Control (MAC) and Physical Layer (PHY) specifications, Standard, IEEE, 1999.

2. IEEE 802.11g WG, Part 11-Amendment 4: Further Higher-Speed Physical Layer Extension in the $2.4 \mathrm{GHz}$ Band, 2003

3. C. E. Perkins, E. M. Royer, and S. R. Das, “Ad hoc on-demand distance vector routing," Internet Draft, 2002.

4. C. E. Perkins, E. M. Royer "Quality of service for ad hoc on-demand distance vector routing". IETF Internet Draft.

5. J. Hsu, S. Bhatia, M. Takai, R. Bagrodia, M. Acriche, "Performance of Mobile Ad Hoc Networking Routing Protocols in Realistic Scenarios," MILCOM '03 (Boston, MA, October 2003).

6. A. Agarwal and W. Wang, "Statistical Analysis of the Impact of Routing in MANET Based on Real-Time Measurements," ICCCN'05, San Diego , California , USA, October, 2005.

7. L. Chen, W. B. Heinzelman "QoS-Aware Routing Based on Bandwidth Estimation for Mobile Ad Hoc Networks" IEEE Journal on Selected Areas in Communications, March 2005.

8. C. Chaudet, I. Guérin Lassous - "BRuIT - Bandwidth Reservation under InTerferences influence" - In Proceedings of European Wireless 2002 (EW2002) - February 2002 Florence, Italy.

9. K. Xu, K. Tang, R. Bagrodia, M. Gerla, and M. Bereschinsky, "Adaptive Bandwidth Management and QoS provisioning in Large Scale Ad Hoc Networks," in Proceedings of MilCom. Boston, Massachusetts,USA: IEEE, Oct. 2003.

10. M Kazantzidis, "End-to-end versus explicit feedback measurement in 802.11 networks," Technical Report $N^{\circ} 010034$ UCLA Computer Science WAM Lab,. 2001.

11. S. H. Shah, Kai Chen, Klara Nahrstedt, "Dynamic Bandwidth Management for Single-hop Ad Hoc Wireless Networks", ACM/Kluwer Mobile Networks and Applications (MONET) Journal, Special Issue on Algorithmic Solutions for Wireless, Mobile, Ad Hoc and Sensor Networks, vol. 10, num. 1, 2005.

12. The Network Simulator - NS-2: http://www.isi.edu/nsnam/ns/ 